\title{
Von Lebensstilen zu kulturellen Präferenzen - Ein Vorschlag zur theoretischen Neuorientierung ${ }^{1}$
}

\author{
Von Jörg Rössel
}

\section{Einleitung}

Die theoretische Diskussion über Sozialstrukturanalyse und soziale Ungleichheit in den vergangenen beiden Jahrzehnten ist in hohem Maße durch Fragen nach der Entstrukturierung der Klassengesellschaft, der Individualisierung von Lebensläufen und der Pluralisierung von Lebensstilen geprägt worden (Berger 1986; Hradil 1987; Beck 1986; Müller 1992; Schulze 1992, Bögenhold 1994). Häufig wurde dabei unterstellt, daß die Bedeutung klassen- und schichtbezogener materieller sozialer Ungleichheiten für das Alltagsleben der Menschen und ihr soziales Handeln abgenommen habe. Daher wurde vorgeschlagen, die Sozialstrukturanalyse stärker auf begriffliche Konzepte zu begründen, die näher am tatsächlichen sozialen Handeln der Menschen liegen (Hradil 1987; Lüdtke 1989). Zwei Begriffe wurden in prominenter Weise diskutiert: einerseits das Konzept der sozialen Milieus, andererseits aber vor allem der Lebensstilbegriff, der in den vergangenen 20 Jahren eine Hochkonjunktur in den Sozialwissenschaften erlebt hat. ${ }^{2}$ Vor allem dieser Begriff soll die relative Abkoppelung der spezifischen Muster sozialen Handelns von materiellen Bedingungen erfassen (Bögenhold 1994: 449; Hradil 2001). Dieses Konzept soll hier skizziert und seine Leistungskraft zusammenfassend dargestellt werden (Abschnitt 2.1.). Dabei wird die These vertreten, daß das Lebensstilkonzept in ein theoretisches Modell der Erklärung sozialen Handelns integriert werden sollte. Die bisherige deskriptive Orientierung des Lebensstilbegriffs führt dazu, daß sich Erklärungen sozialen Handelns auf der Basis von Lebensstiltypologien nicht wesentlich von der vielfach kritisierten Variablensoziologie unterscheiden (Esser 1996), die sich mit der Berechnung von statistischen Korrelationen zwischen Variablen begnügt. Auch hinter den neueren theoretischen Entwicklungen im Bereich der eher klassischen Sozialstrukturanalyse, die ausgesprochen systematisch Klassenanalyse und soziologische Erklärungen verknüpfen, bleibt die Lebensstilforschung damit zurück (Becker 2000; Wright 1997; Breen/Goldthorpe 1997). Hier wird daher vorgeschlagen, den Begriff der Lebensstile, der sich auf manifestes Verhalten bezieht, durch ein Konzept kultureller Präferenzen zu ersetzen. Im Anschluß an Theorien, die soziales Handeln als zielgerichtet und durch Opportunitässtrukturen beschränkt konzipieren (Elster 1989, Franz 1986), können diese kulturellen Präferenzen von Menschen unter Berücksichtigung der jeweiligen Handlungsrestriktionen das Verhalten von Personen erklären.

Die Fruchbarkeit dieser These wird hier in einer empirischen Untersuchung der Determinanten der Häufigkeit der Ausübung von acht verschiedenen Freizeitpraktiken und drei Indikatoren zum Besitz von Konsumgütern verdeutlicht. Die zugrundeliegenden Daten werden in Abschnitt 3 erläutert. Unter Verwendung der vorliegenden Daten können einerseits die kulturellen Präferenzen der Befragten im Anschluß an die von Gerhard Schulze formulierten

1) Für hilfreiche Kommentare und Kritik möchte ich mich bei Jürgen Gerhards, Matthias Junge und den Gutachtern der Sozialen Welt bedanken.

2) Eine Analyse der Datenbanken Solis und Foris ergab, daß Publikationen, die den Begriff Lebensstil enthalten, von den späten siebziger bis zu den späten achtziger Jahren ungefähr 3 Promille aller enthaltenen Publikationen ausmachen. Erst seit den späten achtziger Jahren ist dieser Anteil auf ca. 10 Promille angestiegen und auf diesem Niveau auch durch die ganzen neunziger Jahre hindurch verblieben. Mehr oder weniger parallel zu dieser Entwicklung sind in den achtziger Jahren die Begriffe Klasse und Schicht zunehmend aus sozialwissenschaftlichen Publikationen verschwunden und haben sich in der Häufigkeit ihrer Verwendung an den Begriff der Lebensstile angenähert. 
alltagsästhetischen Schemata operationalisiert werden und andererseits auch eine Reihe von Handlungsrestriktionen berücksichtigt werden. Insgesamt stützen die empirischen Analysen die Fruchtbarkeit der vorgeschlagenen Vorgehensweise (Abschnitt 4). Eine Zusammenfassung und weiterführende Diskussion der Ergebnisse wird in Abschnitt 5 präsentiert.

\section{Theoretische Grundlagen}

\subsection{Die Lebensstildebatte}

Die neuere Sozialstrukturanalyse hat sich mit einer Kritik an der Erklärungsleistung der Konzepte Klasse und Schicht profiliert und konstituiert. Autoren aus unterschiedlichen theoretischen Richtungen vertreten die These, daß die Zugehörigkeit zu sozioökonomischen Schichten oder Klassen die Identität, die Mentalität und das Handeln von Menschen in gegenwärtigen Gesellschaften nur noch schwach prägen (Beck 1986; Schulze 1992; Lüdtke 1989; Hörning/ Michailow 1990). In der Literatur werden vor allem zwei Gründe für die Annahme einer Abkoppelung der lebensweltlichen Mentalitäten und Mustern des sozialen Handels von Schichten und Klassen genannt: Erstens hat die allgemeine Wohlstandssteigerung, die Vergrößerung der Freizeit und eine gewaltige Explosion von marktvermittelten Angeboten dazu geführt, daß die einzelnen Menschen in höherem Maße ihr eigenes Leben nach ihren jeweiligen Wünschen gestalten können (Pierenkemper 1987; Beck 1986; Hradil 1987; Lüdtke 1989; Schulze 1992). Konzepte wie Klasse und Schicht, die vor allem auf die Ausstattung von Individuen und Haushalten mit bestimmten Ressourcen abzielen, seien daher nicht mehr adäquat für die Erfassung gegenwärtiger Strukturen sozialer Ungleichheit. Neben dieser Verbreiterung der materiellen Möglichkeiten hat in modernen Gesellschaften zweitens ein unter anderem durch die Bildungsrevolution induzierter Wertewandel stattgefunden, in dem Pflichtwerte und materialistische Orientierungen teilweise durch Werte der Selbstverwirklichung und der individuellen Freiheit verdrängt worden sind (Inglehart 1989; Klages 1984). Damit stehen den Individuen nicht nur in materieller Hinsicht mehr Optionen offen, sondern sie sind bei der Entwicklung und Gestaltung ihrer kulturellen Werte und Präferenzen unabhängiger von normativen Beschränkungen und klassenspezifischen Traditionen geworden.

Während vor allem Ulrich Beck aus diesen Entwicklungen die Entstehung einer individualisierten Gesellschaft gefolgert hat (Beck 1986), gehen die Autoren im Bereich der Lebenstilforschung davon aus, daß es zu neuen Typen von Vergemeinschaftung zwischen den Menschen gekommen ist (vgl. Meyer 2001: 260 - 261). Gerade dann, wenn die Menschen in größerer Unabhängigkeit von äußeren Beschränkungen ihren Präferenzen folgen können, bilden sich neue Formen von Vergesellschaftung und Vergemeinschaftung heraus. Diese werden auf der Basis der jeweiligen Vorlieben von den Menschen frei gewählt (Schulze 1992). Die Strukturierung des Handelns erfolgt dann nicht durch die Zuordnung von Personen zu Klassen, sondern durch die Zugehörigkeit zu kulturell relativ homogenen Milieus, die durch spezifische Lebensstile gekennzeichnet sind. Der Begriff der Lebensstile steht dabei für relativ stabile Muster des sozialen Handelns von Individuen (Lüdtke 1995: 10; Schulze 1992: 103). ${ }^{3}$ Milieuspezifische Lebensstile haben, so die zugespitzte These in dieser Diskussion, die Klassenlage und andere soziodemographische Variablen in ihrer Erklärungsleistung von sozialem Handeln ersetzt (Hartmann 1999: 11; zusammenfassend Zerger 2000).

3) Dabei finden sich innerhalb der Literatur zum Teil sehr unterschiedliche Definitionen und Operationalisierungen von Lebensstilen. Während z. B. Lüdtke in seiner Definition und Operationalisierung des Begriffs auf die Handlungsebene fokussiert (Lüdtke 1989; 1995), integriert Spellerberg in das Konzept auch die Lebensziele der Befragten (Spellerberg 1996) und bei Otte (2000) finden sich darüber hinaus noch Hinweise auf die Ressourcenausstattung des Haushaltes (vgl. für einen Überblick Hartmann 1999: 15 - 48 und $89-125)$. 
Eine moderatere Variante des Lebensstilkonzepts versucht Klassenlage und milieuspezifische Lebensstile nicht als alternative Erklärungsmuster zu betrachten, sondern miteinander zu verbinden. Klassen- und schichtspezifische Verhaltensweisen werden in dieser Vorstellung durch kulturell relativ homogene Milieus, die durch spezifische Lebensstile geprägt sind, differenziert. Das Konzept der Lebensstile berücksichtigt die Tatsache, daß nicht alle Mitglieder einer Klasse bzw. einer Schicht sich in ihrem Alltag ähnlich verhalten, sondern daß es - vor allem in den wohlhabenden Mittelklassen - eine erhebliche Spannbreite von Lebensstilen gibt (Spellerberg 1996). Die Lebensstile selbst sind aber durch sozioökonomische und soziodemographische Bedingungen bestimmt (Konietzka 1995; Schulze 1992; Hartmann 1999; Bourdieu 1982). Die vorhandenen empirischen Studien zeigen, daß Lebensstile - in welcher Form sie auch immer gemessen werden - mit Alter, Bildung, Geschlecht und sozioökonomischer Lage (die Reihenfolge gibt die relative Höhe der statistischen Korrelationen an) korrelieren (Spellerberg 1996).

Eines der Hauptprobleme der Lebensstilforschung ist die zugrundeliegende These, daß das soziale Handeln der Menschen in vergangenen Gesellschaftsperioden allein durch eine vertikale Klassen- bzw. Schichtstruktur determiniert war. Erst durch den zunehmenden Massenwohlstand und den Wertewandel sei dieser enge Konnex aufgebrochen worden (Schulze 1992: 532 - 535; Müller-Schneider 1994). Erstens liegen innerhalb der Soziologie nur sehr wenige Studien vor, die tatsächlich eine angemessene, longitudinale Perspektive einnehmen, um damit diese These untermauern zu können, zweitens wird in der sozialhistorischen Forschung immer deutlicher, daß auch in der Vergangenheit Klassen oder Schichten keine homogenen Milieuzugehörigkeiten und Lebensstile aufwiesen (Adam 1999, Kocka 1990; Spree 1997). ${ }^{4}$ So kann z. B. Adam nachweisen, daß in einer der Hochburgen der sozialdemokratischen Arbeiterbewegung in Deutschland klassische Organisationen der Arbeiterbewegung keineswegs klassenmäßig homogen zusammengesetzt waren (Adam 1999). Dies gilt auch für die Wählerschaft der SPD (Sperber 1997). Auch im Hinblick auf die Vielfalt von Lebensstilen behauptet Spree auf der Basis einer detaillierten Analyse von Haushaltsrechnungen, daß schon im Deutschen Kaiserreich und der Weimarer Republik Konsumstile und Klassenzugehörigkeiten keineswegs deckungsgleich waren (Spree 1997). Sollten sich diese Ergebnisse erhärten, dann könnte die Ersetzung des Klassen- durch das Lebensstilkonzept nicht mehr mit dem Verweis auf historische Wandlungsprozesse begründet werden. Denn es würde deutlich, daß die Begriffe Klasse, Milieu und Lebensstil analytisch zu trennen sind und die entsprechenden Phänomene schon in historisch relativ lange zurückliegenden Phasen der kapitalistischen Industriegesellschaft empirisch relativ unabhängig voneinander waren. Dann ist aber die rein deskriptive Frage, ob die Sozialstruktur fortgeschrittener Gesellschaften besser durch Klassen- oder Schichtkonzepte auf der einen Seite oder durch Milieu- und Lebensstilbegriffe auf der anderen Seite beschrieben werden kann, obsolet. Man muß davon ausgehen, daß die Klassen- und Milieuzugehörigkeit und der jeweilige Lebensstil historisch schon relativ lange partiell voneinander entkoppelt waren. Damit sind die Begriffe in der beschreibenden Analyse von Gesellschaften tendenziell gleichgeordnet. Das Verhältnis dieser Begriffe sollte nun vor allem theoretisch präziser geklärt werden. Dafür muß freilich ein anderer Bezugspunkt gewählt werden. Versteht man im Anschluß an Max Weber die Soziologie als eine Wissenschaft, die soziales Handeln erklären will, so kann die Meßlatte für den Vergleich von theoretischen Konzepten nur ihre Erklärungskraft im Hinblick auf das soziale Handeln sein. In den folgenden Ausführungen soll daher der theoretische Bezugspunkt für die Verwendung des Lebensstilbegriffs seine Leistungskraft für die Erklärung sozialen Handelns sein.

4) Gerade die häufig als Beleg für die Auflösung von sozialen Milieus zitierte Studie von Mooser (1984) belegt eine im Verlauf des 20. Jahrhundert zunehmende soziale Vernetzung der Arbeiterschaft, die eher für eine Verdichtung des Arbeitermilieus spricht. Dagegen zeigt sie aber den abrupten Bruch in den Institutionen der Arbeiterbewegung, der freilich nicht mit Verweis auf langfristige Modernisierungsprozesse erklärt werden kann, sondern durch die historische Zäsur des 3. Reiches. 
Dabei werde ich mich zuerst den Ergebnissen von empirischen Studien zuwenden, bevor die theoretischen Defizite des Lebensstilkonzepts beleuchtet werden sollen.

Empirisch ist die Erklärungskraft von Lebensstilen und Milieuzugehörigkeiten für das soziale Handeln überaus umstritten (Hartman 1999; Zerger 2000; Uttitz 1985; Müller 1998, MüllerSchneider 1994; Ganzeboom 1987). So vergleicht z. B. Hartmann in seiner wegweisenden Studie die statistische Erklärungskraft der von Schulze konzipierten Lebensstilkonzepte (alltagsästhetische Schemata) mit der Erklärungskraft von traditionellen soziodemographischen Variablen. Dabei dienen ihm 148 Einzelitems, die Präferenzen, Verhaltensweisen und Interessen umfassen, als abhängige Variablen. Es zeigt sich, daß die drei alltagsästhetischen Schemata im Durchschnitt keine größere Varianzaufklärung leisten als die beiden klassischen soziodemographischen Variablen des Alters und der Schulbildung (Hartmann 1999: 213 - 215). Zu ähnlichen Resultaten gelangt Zerger in einem Vergleich der statistischen Erklärungskraft der sogenannten Sinus-Milieus und des EGP-Klassenschemas (Zerger 2000). So wichtig derartige Studien für die Einschätzung der statistischen Erklärungskraft von theoretischen Konzepten sein können, so unbefriedigend bleiben sie freilich aus einer theoretischen Perspektive. Die Feststellung eines statistischen Zusammenhangs zwischen einer Lebensstilvariable oder einer soziodemographischen Variable auf der einen Seite und einem bestimmten Typus sozialen Handelns auf der anderen Seite bleibt letztlich nur eine statistische Korrelation und keine Erklärung (vgl. Esser 1996). Eine Erklärung liegt erst dann vor, wenn wir angeben können, warum Personen, die zu einer bestimmten Klasse gehören, diese oder jene Aktivität häufiger als Personen aus einer anderen Klasse ausüben: „A statistical effect of a class variable in contexts like these is essentially an indicator of our inability to specify properly the underlying explanatory mechanisms. The worse we do in specifying and incorporating the actual generative mechanisms into the statistical model, the stronger the effect of the class variable will appear to be“ (Hedström/Swedberg 1998: 11). Die Ausführungen von Hedström und Swedberg gelten in gleichem Maße auch für die Zuordnung von Personen zu sozialen Milieus, Schichten, Lebensstilgruppen oder anderen Klassifikationen bzw. Typologien. Die Korrelationen zwischen diesen soziologischen Gruppenbildungen auf der einen Seite und bestimmten Formen sozialen Handelns auf der anderen Seite erreichen nicht den Status von theoretischen Erklärungen, wenn der soziale Mechanismus, der die beiden Variablen miteinander verbindet, nicht angegeben werden kann. Daher liegt die Schlußfolgerung nahe, daß die rein empirische Überprüfung der Leistungsfähigkeit von Lebensstilkonzepten durch die Bezugnahme auf eine theoretische Erklärung ergänzt werden muß. Es muß also im nächsten Schritt eine Integration des Lebensstilkonzepts in ein theoretisches Erklärungsmodell sozialen Handelns vorgenommen werden.

\subsection{Handeln als beschränkte Wahl}

Die Überprüfung der empirischen und theoretischen Relevanz von Lebensstilkonzepten für die Erklärung sozialen Handelns leidet bisher darunter, daß diese nicht mit theoretischen Konzepten zur Erklärung sozialen Handelns verbunden worden sind. ${ }^{5}$ Hier soll an ein theoretisch relativ offenes Handlungsmodell angeknüpft werden, das die Handlungen von Akteuren erstens als zielgerichtet begreift, zweitens aber davon ausgeht, daß die Handlungsalternativen eines Akteurs durch spezifische Handlungsressourcen und -restriktionen beschränkt sind. Diese Vor-

5) Eine Ausnahme machen hier die Arbeiten von Hartmut Lüdtke, der explizit an die Theorie rationalen Handelns für die Erklärung der Herausbildung von Lebensstiltypen anknüpft (Lüdtke 1989, 1990; 1995). Freilich bleibt diese theoretische Verbindung eher metaphorisch. Dies zeigt sich z. B. in seiner empirischen Überprüfung der Erklärungskraft von Präferenzen und Handlungsrestriktionen für die Herausbildung von Lebensstilen (Lüdtke 1990). Die von ihm verwendeten Variablen lassen sich keineswegs ohne weiteres aus der Theorie rationalen Handelns ableiten und der kausale Mechanismus, der die jeweiligen Präferenzen und Handlungsrestriktionen mit den jeweiligen Lebensstilen verbindet, bleibt letztlich im Dunkeln. 
stellung menschlichen Handelns könnte man als kleinsten gemeinsamen Nenner handlungstheoretischer Ansätze in der Soziologie bezeichnen (Elster 1989; Franz 1986). ${ }^{6}$ In diesem Modell wird menschliches Handeln als das Ergebnis von zwei aufeinanderfolgenden Filtermechanismen begriffen (Elster 1989: 13; vgl. Opp 1999: 173). Ein erster Filterprozeß strukturiert die Menge aller möglichen Handlungsalternativen nach ihrer Zugänglichkeit für den Akteur, man könnte hier von einer Opportunitätsstruktur sprechen. Diese Opportunitätsstruktur wird durch das Verhältnis von zwei Größen gebildet: erstens den Kosten für eine spezifische Handlungsalternative, und zweitens den Ressourcen, über die der Akteur verfügt. Erst im zweiten Filterprozeß greifen nun die Vorlieben des Akteurs, so daß er aus der gegebenen Opportunitätsstruktur diejenigen Alternativen auswählt, die seinen persönlichen Vorlieben bzw. seinen Präferenzen entsprechen.

Das hier nur kurz eingeführte Handlungsmodell erlaubt die Formulierung eines sozialen Mechanismus für die Auswahl von Handlungsalternativen. Wir können erstens davon ausgehen, daß Akteure zielgerichtet handeln, daß sie also Handlungen auswählen werden, von denen sie annehmen können, daß sie ihren Präferenzen entsprechen. Dies geschieht aber zweitens im Rahmen einer Opportunitätstruktur, die eine Einschränkung der Handlungsalternativen für den Akteur bedeutet. Eine Person mit einer Vorliebe für klassische Musik kann z. B. aus einer breiten Palette von Handlungsangeboten wählen, um diesem Musikgeschmack zu folgen: vom Hören einer CD im heimischen Wohnzimmer, über den Besuch eines Konzertes auf den billigsten Plätzen bis hin zum privaten Engagement eines international rennomierten Orchesters gibt es eine unendliche Vielzahl von Möglichkeiten, klassische Musik zu konsumieren. Welche dieser Alternativen tatsächlich gewählt wird, hängt also nicht nur von den Vorlieben der Person ab, sondern von ihrer Opportunitätsstruktur - also den Kosten der verschiedenen Optionen auf der einen Seite und ihren eigenen Ressourcen auf der anderen Seite. Insofern muß die Bedeutung von Lebensstilen oder kulturellen Vorlieben aus theoretischer Perspektive in gewissem Maße eingeschränkt werden. Sie werden vor allem in Entscheidungen, deren Konsequenzen mit geringen Kosten behaftet sind, von großer Relevanz sein. Dagegen wird ihre explanatorische Bedeutung in Handlungssituationen mit hohen Kosten von geringerer Bedeutung sein (Quandt/ Ohr 2004; Mensch 2000; Zintl 1989). So hat Diekmann zeigen können, daß das Umweltbewußtsein von Personen vor allem in Situationen mit niedrigen Kosten das faktische Umweltverhalten beeinflußt, dagegen in Hochkostensituationen keine Relevanz besaß (Diekmann 1996). In den letztgenannten Situationen sind vor allem die Handlungsrestriktionen für die Erklärung zentral. Daher kann man auch für Lebensstile bzw. kulturelle Präferenzen einen relativ großen Einfluß nur in Niedrigkostensituationen vermuten, während sie in Hochkostensituationen eine geringere Erklärungskraft aufweisen. Entscheidung im letztgenannten Situationstyp werden stärker durch die Verfügung über materielle Ressourcen bestimmt werden, also durch Merkmale, die in höherem Maße an Klasse und Schicht gebunden sind.

Will man an eine derartige handlungstheoretische Perspektive anknüpfen, dann müßte der Lebensstilbegriff durch das Konzept der kulturellen Präferenzen der Personen ersetzt werden. Da hier allerdings keine Engführung auf die Theorie rationalen Handelns vorgenommen werden soll, könnte man auch von kulturellen Dispositionen der Akteure oder schlicht von deren Vorlieben sprechen. Betrachtet man auf die Ursprünge der Lebensstildiskussion vor

6) Da es hier nicht um die Argumentation für oder gegen eine bestimmte Handlungstheorie geht, sondern generell um die Verbindung des Lebensstilkonzepts mit einer Handlungstheorie, wird diese theoretisch ausgesprochen breit anschlußfähige handlungstheoretische Konzeption gewählt. Daher wurde auch bewußt auf die Ergänzung durch eine Nutzenmaximierungsannahme verzichtet (vgl. Opp 1999: 173). Die Frage, ob man die hier vorgestellte Argumentation an die Theorie des rationalen Handelns (Opp 1999; Esser 2002), stärker an emotionssoziologisch fundierte akteurtheoretische Konzepte (Collins/Hanneman 1998; McKinnon 1994) oder schließlich an den symbolischen Interaktionismus (Joas 1992; Fine 1995) anknüpft, scheint mir für die hier diskutierte Argumentation von untergeordneter Bedeutung zu sein. 
allem in den Arbeiten von Pierre Bourdieu, so fokussiert der hier gemachte theoretische Vorschlag stärker auf die Begriffe Geschmack und Habitus und weniger auf seinen Lebensstilbegriff (Bourdieu 1982; Müller 1986). Diese Konzeptualisierung erlaubt die Unterscheidung zwischen zwei verschiedenen Kausalzusammenhängen zwischen strukturellen Bedingungen und Präferenzen von Akteuren. Erstens kann untersucht werden, wie die sozialstrukturelle Lage die kulturellen Präferenzen bzw. Dispositionen einer Person prägt. Darüber hinaus ist zweitens auch die Chance gegeben, die Realisierung von Präferenzen unter Berücksichtigung von strukturellen Handlungsrestriktionen $\mathrm{zu}$ erforschen. Soziologische Studien, die unmittelbar den statistischen Zusammenhang zwischen Sozialstruktur auf der einen Seite und Lebensstilen auf der anderen Seite untersuchen, können diese beiden kausalen Mechanismen daher nicht systematisch differenzieren.

Ersetzt man den Lebensstilbegriff durch das Konzept der kulturellen Präferenzen, so steht man freilich vor dem empirischen Problem, daß Lebensstile üblicherweise durch Muster sozialen Handelns - meist Freizeitaktivitäten - operationalisiert werden. Die hier vorgeschlagene Begrifflichkeit würde dagegen eine konsequente Operationalisierung von kulturellen Präferenzen durch die Frage nach Vorlieben und Abneigungen nahelegen. ${ }^{7}$ Dies soll in der folgenden eher explorativen empirischen Studie Berücksichtigung finden.

In diesem Aufsatz sollen die kulturellen Präferenzen der Akteure im Anschluß an die Arbeiten von Gerhard Schulze konzipiert werden. Dieser hat drei Typen von alltagsästhetischen Schemata unterschieden, die eine Vielzahl von gesellschaftlich produzierten ästhetischen Zeichen auf eine geringe Zahl von Grundbedeutungen beziehen und stellen damit einen plausiblen Ausgangspunkt für die Bestimmung von kulturellen Präferenzen dar. Sie sind Resultat kollektiver und individueller Definitionsanstrengungen, bei denen bestimmte Zeichen als trivial, unanständig und kitschig definiert werden und andere als kultiviert und auratisch (Schulze 1992: 137). In seiner empirischen Studie kristallisieren sich drei solcher alltagsästhetischer Schemata heraus: das Hochkultur-, das Trivial- und das Spannungsschema (Schulze 1992: 142 - 157). Während die Alltagsästhetik laut Schulze noch vor wenigen Jahrzehnten eindimensional durch den Gegensatz zwischen traditioneller Hochkultur auf der einen Seite und Trivialkultur auf der anderen Seite geprägt war, hat die Entwicklung des Spannungsschemas zu einer Mehrdimensionalität des Raums der Alltagsästhetik geführt (Müller-Schneider 1994; 1996). Damit ist eine Form der Alltagsästhetik gemeint, die sich an Schnelligkeit, Körperlichkeit und Action orientiert. Typische Beispiele für das Spannungsschema sind Rock- und Popmusik, Kinobesuch, Actionfilme und modische Formen des Tanzens (Schulze 1992: 153 - 154). Diese drei alltagsästhetischen Schemata können als kulturelle Präferenzen für bestimmte Klassen von sozialen Handlungen betrachtet werden.

Zusammenfassend kann festgehalten werden, daß das Konzept der Lebensstile mit einer theoretischen Erklärung sozialen Handelns verbunden werden kann. Allerdings muß der Lebensstilbegriff dabei durch ein Konzept kultureller Präferenzen ersetzt werden, das nicht die faktischen Muster sozialen Handelns bezeichnet, sondern die typischen Vorlieben für bestimmte Klassen sozialen Handelns meint. Eine derartige Vorgehensweise eröffnet erstens die Möglichkeit, die Erklärungskraft kultureller Präferenzen für bestimmte Formen sozialen Handelns unter Berücksichtigung der Opportunitätsstruktur der Akteure zu untersuchen. Zudem kann auf dieser begrifflichen Grundlage zweitens auch die Prägung von kulturellen Präferenzen bzw. Dispositionen durch sozialstrukturelle Bedingungen untersucht werden. Damit wäre eine vollständige Verbindung zwischen den kulturellen Präferenzen und einer theoreti-

7) Die Operationalisierung grundsätzlicher kultureller Präferenzen oder Lebensstile mit Hilfe von Freizeitpraktiken mag eine gangbare Alternative sein, wenn keine direkten Geschmacksindikatoren vorliegen und eine deutlich andere Art von Verhalten erklärt werden soll (vgl. Gerhards/Rössel 2002). 
schen Erklärung sozialen Handelns unter Berücksichtigung struktureller Bedingungen erreicht erreicht. Zudem bleibt die Grundmotivation für die Einführung des Lebensstilbegriffs auch im Konzept der kulturellen Präferenzen erhalten: die Eigenlogik der kulturellen und symbolischen Sphäre der Gesellschaft kann ergänzend zu der Verteilung von materiellen Ressourcen berücksichtigt werden. Der empirischen Umsetzung dieser Konzeption werden sich die nächsten beiden Abschnitte widmen.

\section{Daten und Methoden}

Die folgende empirische Studie basiert auf zwei schriftlichen Umfragen in Leipzig, wobei die erste im Jahr 2000 im Auftrag der Oper Leipzig und die zweite im Jahr 2002 in einem sozial heterogenen Stadtteil der Stadt Leipzig im Kontext eines stadtsoziologischen Forschungsseminars durchgeführt wurde. Beide wurden im Rahmen eines größeren Forschungszusammenhangs zum Thema Sozialstruktur, soziale Milieus und Lebensstile konzipiert. Der relativ spezielle Inhalt und Auftraggeber der ersten Umfrage hat zu einer eher niedrigen Rücklaufquote und zu einer verzerrten Stichprobe geführt. Von insgesamt 3000 angeschriebenen Personen - die auf der Basis einer Zufallsstichprobe ausgewählt wurden - haben nur 630 Personen geantwortet, also wurde nur eine Rücklaufquote von $21 \%$ erreicht. Zudem ist die Stichprobe verzerrt, da Opernbesucher überrepräsentiert sind. Während laut Bürgerumfrage in Leipzig nur ein Anteil von $24 \%$ der Leipziger Bevölkerung in jedem Jahr mindestens einmal die Oper Leipzig besucht (zusätzlich $20 \%$ seltener als einmal im Jahr), waren dies unter den Befragungsteilnehmern $43 \%$ (Stadt Leipzig 2003: 81). Insofern liegt also ein Datensatz mit einer deutlichen Überrepräsentation von höher gebildeten Personen vor, die vermutlich eine stärkere Orientierung am Hochkulturschema aufweisen (vgl. Tabelle 1). Die asymmetrische Stichprobe ist freilich für die hier behandelte Fragestellung unproblematisch, da es sich nicht um die Schätzung von Parametern der Grundgesamtheit handelt, sondern um die Überprüfung von Zusammenhangshypothesen. Diese können auch an verzerrten oder willkürlichen Stichproben getestet werden (Dieckmann 1995: 329). In der anderen Befragung konnte eine Rücklaufquote von $37 \%$ erreicht werden und der Datensatz weist eine deutlich geringere Verzerrung auf (vgl. Tabelle 1).

Tabelle 1: Alters- und Bildungsstruktur der Befragten in \% im Vergleich zur Grundgesamtheit

\begin{tabular}{lccc}
\hline & $\begin{array}{c}\text { Mikrozensus } \\
\mathbf{2 0 0 0}^{\mathbf{a}}\end{array}$ & $\begin{array}{c}\text { Opernumfrage } \\
\mathbf{2 0 0 0}\end{array}$ & $\begin{array}{c}\text { Stadtteilumfrage } \\
\mathbf{2 0 0 2}\end{array}$ \\
\hline Alter & 12 & & \\
$18-24$ & 17 & 16,9 & 15,4 \\
$25-34$ & 18 & 23,7 & 29,6 \\
$35-44$ & 16 & 20,8 & 13,4 \\
$45-54$ & 17 & 13,2 & 13,3 \\
$55-64$ & 21 & 15,4 & 11,1 \\
$65-$ & & 10,2 & 14,9 \\
\hline Bildung & 31,14 & 7,6 & 23,3 \\
max. Volks-/Hauptschule & 43,18 & 41,1 & 27,4 \\
Mittlere Reife/POS & 26,92 & 51,3 & 49,3 \\
Abitur & & & \\
\hline
\end{tabular}

a. Die Angaben stammen aus Stadt Leipzig $(2001 ; 2003)$. 
Mit Hilfe der vorliegenden Datensätze kann also die Fragestellung des Aufsatzes empirisch untersucht werden. Dabei soll allerdings die Relevanz der kulturellen Präferenzen bzw. Dispositionen für die Erklärung des Handelns unter Berücksichtigung von Handlungsrestriktionen im Mittelpunkt stehen. Die Prägung dieser Präferenzen durch die strukturelle Lage der Akteure wurde schon vielfach zum Thema empirischer Untersuchungen gemacht (Bourdieu 1982). Es wurde hier versucht, die kulturellen Vorlieben der Akteure möglichst sparsam $\mathrm{zu}$ operationalisieren. Eines der großen Probleme der bisherigen Lebensstilforschung besteht in der Notwendigkeit, die Lebensstile der befragten Personen auf der Basis einer Vielzahl von Indikatoren zu bilden (vgl. Otte 2000). Demgegenüber sollte hier in explorativer Weise versucht werden, die kulturellen Präferenzen der Befragten, die hier im Anschluß an Schulzes alltagsästhetische Schemata konzipiert werden, mit Hilfe von möglichst wenigen Indikatoren zu operationalisieren. Dabei fiel die Wahl auf die Musikpräferenzen der Personen als Basisindikatoren für deren kulturelle Orientierung. Die musikalischen Vorlieben nehmen eine relativ zentrale Rolle im Geschmack einer Person ein. Pierre Bourdieu z. B. sieht im musikalischen Geschmack eine nahezu unfehlbare Dokumentation der Klassenzugehörigkeit einer Person (Bourdieu 1982: 41). Zudem sind auch in Schulzes eigener Studie die musikalischen Vorlieben die stärksten Indikatoren für die Orientierung an einem der drei alltagsästhetischen Schemata (Schulze 1992: 621 - 628). Insofern scheinen mit den Musikpräferenzen große Bereiche der kulturellen Vorlieben von Akteuren abgedeckt zu sein (Gebesmair 2001). Diese Annahme wird in der folgenden empirischen Studie an musikfernen Aktivitäten und am Besitz von Konsumgütern überprüft.

Hier wurde der Versuch gemacht, die drei kulturellen Präferenzen auf der Basis von nur acht Musikvorlieben zu konstruieren: Pop, Rock und Techno wurden zum Spannungsschema, Klassik und Oper zum Hochkulturschema und Volksmusik, Blasmusik und Schlager zum Trivialschema zusammengefaßt. Dabei wurde in den Erhebungen nicht gefragt, wie häufig diese Musikrichtungen gehört werden, sondern nach der Vorliebe für diese Arten von Musik. Entgegen der Annahme, daß diese beiden Fragetypen mehr oder weniger das gleiche Resultat erzeugen, liegt die statistische Korrelation zwischen der Präferenz für Opernmusik und der Häufigkeit des Hörens dieser Musikrichtung nur bei 0,47. Dies entspricht der in psychologischen Studien häufig festgestellten, relativ niedrigen Korrelation zwischen allgemeinen Einstellungen und Verhalten (Frey/Stahlberg/Gollwitzer 1993). Insofern ist eine Erklärung von spezifischen Handlungsmustern auf der Basis von kulturellen Präferenzen auch keineswegs tautologisch, da neben den Vorlieben der Akteure auch deren Handlungsrestriktionen berücksichtigt werden müssen. ${ }^{8}$

Die hier vorgenommene Operationalisierung der drei alltagsästhetischen Schemata erzeugt Resultate, die inhaltlich den Ergebnissen von Gerhard Schulze sehr nahe kommen. Um die Kriteriumsvalidität der Operationalisierung zu überprüfen, wurde die Korrelation der alltagsästhetischen Schemata mit Alter und Bildung berechnet, die in Schulzes Modell zentrale situative Milieukennzeichen sind und in charakteristischer Weise mit den alltagsästhetischen Schemata korrelieren. Die Ergebnisse sind in Tabelle 2 dargestellt. Dort finden sich auch die vergleichbaren Resultate von Schulze für Nürnberg, Lechner (1998) für Chemnitz und von Hartmann (1999) für die Stadt Köln. Es zeigt sich deutlich, daß die hier vorgenommene Operationalisierung der drei alltagsästhetischen Schemata ähnliche Resultate erzeugt wie in anderen Studien. Dies spricht dafür, daß man mit Hilfe der musikalischen Präferenzen den Kerngehalt der alltagsästhetischen Schemata erfassen kann. Zudem wird im Vergleich zu

8) Freilich ist davon auszugehen, daß zwischen den kulturellen Vorlieben bzw. Dispositionen von Personen und den strukturellen Rahmenbedingungen ihres Handelns eine relativ gute Passung existiert, da die jeweiligen Präferenzen von diesen Strukturbedingungen geprägt wurden (Bourdieu/Wacquant 1996: 124 - 175). 
Lechners Studie über Chemnitz deutlich, daß in Ostdeutschland der Zusammenhang zwischen Bildung und den alltagsästhetischen Schemata niedriger ist als in Westdeutschland. Hier scheint ein Spezifikum der ostdeutschen kulturellen Orientierung deutlich zu werden (vgl. Lechner 1998: 270 - 272). Damit wird natürlich auch ersichtlich, daß die kulturellen Vorlieben von Personen in starkem Maße von ihrer sozialstrukturellen Position geprägt werden, auch wenn dies hier nicht explizit untersucht werden soll.

Tabelle 2: Alltagsästhetische Schemata, Bildung und Alter

\begin{tabular}{lrr|lr|lr|lr|rr}
\hline & \multicolumn{2}{l|}{ Nürnberg 1985 } & \multicolumn{2}{l|}{ Köln 1995 } & \multicolumn{2}{l|}{ Chemnitz 1996 } & \multicolumn{2}{l|}{ Leipzig 2000 } & \multicolumn{2}{l}{ Leipzig 2002 } \\
\hline & Alter & Bildung & Alter & Bildung & Alter & Bildung & Alter & Bildung & Alter & Bildung \\
Hochkultur & 0,05 & 0,45 & - & 0,39 & - & 0,35 & 0,38 & 0,20 & 0,22 & 0,20 \\
Trivial & 0,47 & $-0,50$ & 0,59 & $-0,52$ & 0,47 & $-0,20$ & 0,57 & $-0,26$ & 0,66 & $-0,37$ \\
Spannung & $-0,64$ & 0,25 & $-0,75$ & 0,50 & $-0,60$ & - & $-0,71$ & $-0,02$ & $-0,59$ & 0,24 \\
\hline
\end{tabular}

Entsprechend dem oben skizzierten theoretischen Modell des Handelns sollen neben den kulturellen Präferenzen der Personen, die hier durch die alltagsästhetischen Schemata von Schulze operationalisiert werden, in dieser Studie auch die Opportunitätsstrukturen bzw. die Handlungsrestriktionen der Personen berücksichtigt werden. Hier können wir drei Variablen heranziehen. Erstens die Distanz des Wohnstandortes des Befragten von der Innenstadt, in der sich zahlreiche kulturelle Einrichtungen konzentrieren. Dazu wurde die durchschnittliche Entfernung des jeweiligen Postleitzahlbezirks vom Stadtzentrum berechnet. Diese Variable sollte freilich nur bei denjenigen kulturellen Praktiken relevant sein, die im Stadtzentrum konzentriert sind. Je höher die Entfernung der Wohnung vom Stadtzentrum, desto niedriger sollte hier die Häufigkeit der Ausübung sein. Kulturelle Aktivitäten mit einer dezentralen Angebotsstruktur dürften in der Häufigkeit ihrer Ausübung nicht durch die Distanz des Wohnstandortes vom Stadtzentrum bedingt werden. Zweitens konnten wir einen Index für die Zeitrestriktionen der Befragten auf der Basis ihrer Wochenarbeitszeit und der Zahl ihrer Kinder unter 14 Jahren bilden. Eine solche Proxyvariable weist eine hohe Korrelation mit der tatsächlich frei verfügbaren Zeit auf (Ganzeboom 1987). Je höher die Zeitrestriktionen, desto niedriger sollte die Häufigkeit der Ausübung kultureller Praktiken sein. Drittens verwenden wir noch das Nettoeinkommen pro Kopf im Haushalt (Äquivalenzeinkommen nach der neuen OECD-Skala) als Handlungsrestriktion, da die hier berücksichtigten kulturellen Praktiken mit finanziellen Aufwendungen verbunden sind und daher in der Häufigkeit ihrer Ausübung durch das jeweilige Haushaltsbudget beschränkt sind. Je höher das Haushaltsbudget ist, desto häufiger werden - so die Hypothese - die kulturellen Praktiken ausgeübt. Bei der Analyse der Verfügung über Konsumgüter wird nur diese dritte Handlungsrestriktion berücksichtigt, da vor allem die Verfügung über ökonomische Ressourcen den Besitz von Konsumgütern beeinflussen sollte, auch wenn diese durchaus von Relevanz für den Lebensstil einer Person sind. Generell liegt diese Handlungsrestriktion der klassischen Sozialstrukturanalyse am nächsten, da die Verteilung von materiellen Ressourcen vergleichsweise stark durch die Klassen- oder Schichtzugehörigkeit geprägt wird (Hradil 1999; Zerger 2000). Dagegen sind die beiden anderen Handlungsrestriktionen durch andere Kontexte, wie Erwerbstätigkeit, Familienbildung und räumliche Kontexte strukturiert. Damit wird berücksichtigt, daß die strukturellen Bedingungen des sozialen Handelns zum Teil nicht von der Klassenstruktur geprägt sind.

Schließlich kann die folgende Gruppe von abhängigen Variablen verwendet werden, die alle die Häufigkeit bestimmter Freizeitaktivitäten angeben: Besuch des Konzerthauses (Gewandhaus), Besuch von Ballettvorstellungen, Besuch des Schauspielhauses, Besuch des Mu- 
seums der Bildenden Künste, Besuch von Kneipen und Cafés, Besuch von Kinos, Besuch von Diskos sowie das Hören von Opernmusik in der eigenen Wohnung. Die Häufigkeit des Hörens von Opernmusik in der eigenen Wohnung dient hier als Vergleichsbeispiel. Beim Hören von Opernmusik im eigenen Haushalt sollten alle drei Handlungsrestriktionen nicht greifen, da keine Entfernung von der eigenen Wohnung zu überwinden ist, die Zeitrestriktionen eine geringere Rolle spielen, da man die Musik auch neben anderen Tätigkeiten hören kann und da schließlich auch die finanziellen Kosten geringfügig sind (CD-Spieler haben eine weite Verbreitung, CDs können mehr oder weniger kostenlos aus Stadtbüchereien ausgeliehen werden und schließlich ist als kostengünstigste Alternative das Hören von Opernmusik im Radio zu nennen). Insofern sollten bei der Erklärung der Häufigkeit des Opernmusikhörens im eigenen Haushalt nur die kulturellen Präferenzen relevant sein und keiner der Indikatoren für die Opportunitätsstrukturen.

Zusätzlich kann noch eine weitere Differenzierung unter dieser Gruppe von abhängigen Variablen vorgenommen werden: außer den Kinos und den Diskotheken, die auch an den Stadträndern vorhanden sind, sind alle anderen Aktivitäten in hohem Maße auf das Stadtzentrum konzentriert. Daher sollte die Distanz des Wohnstandortes von der Innenstadt nicht nur beim Opernhören im eigenen Haushalt, sondern auch beim Kinobesuch und beim Diskothekenbesuch keine Rolle spielen. Die Opportunitätsstrukturen sind hier nicht für alle Aktivitäten gleichartig. Schließlich können die kulturellen Praktiken auch in musiknahe (Gewandhaus, Ballett, Diskothekenbesuch, Hören von Opermusik) und musikferne (Schauspiel, Museum, Kino, Kneipe/Café) aufgeteilt werden. Hier wurde die These vertreten, daß die musikalischen Präferenzen einen geeigneten Indikator für die gesamten kulturellen Vorlieben einer Person darstellen könnten. Daher sollte die statistische Erklärungskraft der musikalischen Präferenzen für musikferne und musiknahe kulturelle Praktiken gleich groß sein.

Um die oben erläuterte These, daß kulturelle Präferenzen vor allem in Handlungssituationen mit geringen Kosten von großer explanatorischer Bedeutung sind, zu überprüfen, wurden auch drei Indikatoren für die Verfügung über Konsumgüter als abhängige Variablen berücksichtigt: erstens der Besitz eines Autos, zweitens eine mindestens einwöchige Urlaubsreise pro Jahr und drittens der gelegentliche Konsum von Delikatessen (z. B. Champagner). Damit wurden durchaus Konsumgüter berücksichtigt, die auch für den Lebensstil einer Person von hoher Relevanz sind. Sollte die geäußerte These zutreffen, so sollte bei diesen abhängigen Variablen vor allem die Verfügung über ökonomische Ressourcen zentral sein, während die kulturellen Präferenzen eine untergeordnete Bedeutung für die Erklärung ihres Besitzes haben sollten.

Ob mit Hilfe der hier skizzierten Konzeption die Häufigkeit kultureller Aktivitäten und die Verfügung über Konsumgüter zureichend erklärt werden kann, soll unter Kontrolle von zusätzlichen Variablen überprüft werden. Es wurden dazu drei Kontrollvariablen ausgewählt, Alter, Bildung und Geschlecht, da diese in zahlreichen Studien den größten Einfluß auf die Lebensstilgestaltung haben (Spellerberg 1996; Hartmann 1999). Insofern ergibt sich unter Berücksichtigung dieser Variablen ein besonders strenger Test des vorgeschlagenen Konzepts. Wenn sich in den folgenden Auswertungen noch statistisch signifikante Effekte dieser Kontrollvariablen zeigen, so sind die kulturellen Präferenzen und die Handlungsrestriktionen offensichtlich noch nicht vollständig spezifiziert. Ein Gesamtüberblick über die zu prüfenden Hypothesen findet sich in Tabelle 3. 
Tabelle 3: Hypothesen

$\mathrm{H}_{1}$ : Die berücksichtigten kulturellen Präferenzen und Handlungsrestriktionen erklären die kulturellen Praktiken und die Verfügung über Konsumgüter vollständig.

$\mathrm{H}_{2}$ : Die ausgewählten Kontrollvariablen, Alter, Bildung und Geschlecht, haben keinen statistisch signifikanten Einfluß auf die abhängigen Variablen.

$\mathrm{H}_{3}$ : Die Erklärungskraft der kulturellen Präferenzen ist bei musiknahen und musikfernen kulturellen Praktiken gleich groß. ${ }^{a}$

$\mathrm{H}_{4}$ : Die räumliche Distanz des Wohnortes vom Stadtzentrum ist nur bei Aktivitäten relevant, die vorwiegend im Stadtzentrum ausgeübt werden können.

$\mathrm{H}_{5}$ : Die kulturellen Präferenzen sind insbesondere in Niedrigkostensituationen erklärungskräftig, während in Hochkostensituationen die Handlungsrestriktionen von größerer Bedeutung sind:

$\mathrm{H}_{51}$ : In Situationen, die mit sehr niedrigen Kosten verbunden sind (Opernhören im eigenen Haushalt) sind alle Handlungsrestriktionen für die Erklärung irrelevant.

$\mathrm{H}_{52}$ : In Situationen mit geringen Kosten (alle kulturellen Aktivitäten außer Opernhören im eigenen Haushalt) sind vor allem die kulturellen Präferenzen für die Erklärung von Verhalten relevant, die Handlungsrestriktionen beschränken aber das faktisch ausgeübte Handeln.

$\mathrm{H}_{53}$ : In Situationen mit hohen Kosten (Verfügung über Konsumgüter) sind vor allem die Handlungsrestriktionen für die Erklärung des Verhaltens relevant.

a. Bei dieser Hypothese werden die Hochkostensituationen nicht berücksichtigt, da hier die kulturellen Präferenzen laut Hypothese 5.3 generell einen geringeren Einfluß haben sollten.

Die empirische Untersuchung des statistischen Einflusses der verschiedenen kulturellen Präferenzen und der Opportunitätsstrukturen auf die Häufigkeit der Ausübung bestimmter kultureller Praktiken wurde mit Hilfe von linearen Regressionen berechnet. Da einige abhängige Variablen nur drei (Ballett, Schauspielhaus, Museum) bzw. vier Ausprägungen (Gewandhaus) aufweisen, also nur ordinalskaliert sind, haben wir diese auch mit Hilfe von ordered-probality Modellen untersucht (Greene 1992). Da sich nur geringfügige Unterschiede zu den Resultaten von OLS-Regressionen fanden, wurden hier die Ergebnisse der einfacher nachvollziehbaren linearen Regressionsmodelle dargestellt. Für den Besitz der Konsumgüter mußten allerdings logistische Regressionen berechnet werden, da die abhängige Variable in diesem Fall dichotom codiert ist.

\section{Resultate}

In der empirischen Untersuchung sollen nun die aus der theoretischen Diskussion abgeleiteten Hypothesen getestet werden. Dabei geht es vor allem um die Frage, ob die ausgewählten Freizeitaktivitäten und die Verfügung über Konsumgüter allein auf der Basis von kulturellen Präferenzen und Handlungsrestriktionen erklärt werden können. Dabei wurde (siehe Tabelle 3) die Wirkung der verschiedenen Handlungsrestriktionen und -ressourcen im Hinblick auf die jeweiligen Freizeitaktivitäten und Konsumgüter hin spezifiziert. Die Resultate der empirischen Überprüfung dieser Thesen mit Hilfe von Regressionsmodellen finden sich in Tabelle 4 für das Freizeitverhalten und in Tabelle 5 für die Verfügung über Konsumgüter. 
Tabelle 4: Die Bedeutung von kulturellen Präferenzen und Handlungsrestriktionen für das Freizeitverhalten

\begin{tabular}{|c|c|c|c|c|c|c|c|c|}
\hline Variablen & $\begin{array}{l}\text { Gewand- } \\
\text { haus }\end{array}$ & Ballett & $\begin{array}{l}\text { Schau- } \\
\text { spiel }\end{array}$ & $\begin{array}{l}\text { Muse- } \\
\text { um }\end{array}$ & Kneipe & Kino & Disko & Oper \\
\hline Konstante & $\begin{array}{l}1,17 * * * \\
(3,70)\end{array}$ & $\begin{array}{c}0,32 \\
(1,05)\end{array}$ & $\begin{array}{l}1,29 * * * \\
(3,76)\end{array}$ & $\begin{array}{l}1,21 * * * \\
(3,66)\end{array}$ & $\begin{array}{c}5,36 * * * * \\
(12,18)\end{array}$ & $\begin{array}{c}3,66 * * * * \\
(10,07)\end{array}$ & $\begin{array}{c}3,35 * * * \\
(7,82)\end{array}$ & $\begin{array}{l}4,84 * * * \\
(8,43)\end{array}$ \\
\hline Hochkultur & $\begin{array}{l}0,40 * * * \\
(8,65)\end{array}$ & $\begin{array}{l}0,36^{* * * *} \\
(7,45)\end{array}$ & $\begin{array}{l}0,27 * * * \\
(5,13)\end{array}$ & $\begin{array}{l}0,31 \text { *** } \\
(6,16)\end{array}$ & $\begin{array}{c}-0,02 \\
(-0,43)\end{array}$ & $\begin{array}{c}0,06 \\
(1,42)\end{array}$ & $\begin{array}{l}-0,08^{* *} \\
(-2,10)\end{array}$ & $\begin{array}{l}0,39 * * * \\
(8,08)\end{array}$ \\
\hline Spannung & $\begin{array}{l}-0,15 * * * \\
(-2,72)\end{array}$ & $\begin{array}{c}0,03 \\
(0,49)\end{array}$ & $\begin{array}{c}-0,03 \\
(-0,53)\end{array}$ & $\begin{array}{c}-0,02 \\
(-0,27)\end{array}$ & $\begin{array}{c}0,09 * \\
(1,66)\end{array}$ & $\begin{array}{l}0,15 * * * \\
(2,99)\end{array}$ & $\begin{array}{l}0,27 * * * * \\
(5,50)\end{array}$ & $\begin{array}{l}-0,13^{* *} \\
(-2,11)\end{array}$ \\
\hline Trivialkultur & $\begin{array}{c}-0,07 \\
(-1,32)\end{array}$ & $\begin{array}{l}-0,12 * * \\
(-2,33)\end{array}$ & $\begin{array}{l}-0,14 * * \\
(-2,53)\end{array}$ & $\begin{array}{l}-0,14 * * \\
(-2,47)\end{array}$ & $\begin{array}{l}-0,11 * * \\
(-2,19)\end{array}$ & $\begin{array}{l}-0,16^{* * * *} \\
(-3,39)\end{array}$ & $\begin{array}{c}-0,06 \\
(-1,37)\end{array}$ & $\begin{array}{c}0,07 \\
(1,28)\end{array}$ \\
\hline Distanz & $\begin{array}{l}-0,13 * * * \\
(-2,96)\end{array}$ & $\begin{array}{l}-0,15^{* * * *} \\
(-3,34)\end{array}$ & $\begin{array}{c}-0,08 \\
(-1,64)\end{array}$ & $\begin{array}{l}-0,13 * * * * \\
(-2,79)\end{array}$ & $\begin{array}{l}-0,18 * * * \\
(-4,28)\end{array}$ & $\begin{array}{c}-0,02 \\
(-0,55)\end{array}$ & $\begin{array}{c}0,01 \\
(0,29)\end{array}$ & $\begin{array}{c}0,04 \\
(0,84)\end{array}$ \\
\hline Restriktionen & $\begin{array}{c}0,03 \\
(0,55)\end{array}$ & $\begin{array}{c}-0,05 \\
(-1,10)\end{array}$ & $\begin{array}{c}0,02 \\
(0,30)\end{array}$ & $\begin{array}{c}-0,07 \\
(-1,33)\end{array}$ & $\begin{array}{l}-0,10^{* *} \\
(-2,16)\end{array}$ & $\begin{array}{c}-0,02 \\
(-0,43)\end{array}$ & $\begin{array}{l}-0,12^{* * * *} \\
(-2,96)\end{array}$ & $\begin{array}{c}0,05 \\
(1,11)\end{array}$ \\
\hline Einkommen & $\begin{array}{c}0,08^{*} \\
(1,72)\end{array}$ & $\begin{array}{c}-0,02 \\
(-0,40)\end{array}$ & $\begin{array}{c}-0,08 \\
(-1,41)\end{array}$ & $\begin{array}{c}-0,07 \\
(-1,36)\end{array}$ & $\begin{array}{c}0,05 \\
(1,06)\end{array}$ & $\begin{array}{c}-0,05 \\
(-1,01)\end{array}$ & $\begin{array}{l}-0,10^{* *} \\
(-2,33)\end{array}$ & $\begin{array}{c}-0,06 \\
(-1,12)\end{array}$ \\
\hline Bildung & $\begin{array}{l}0,14 * * * \\
(3,15)\end{array}$ & $\begin{array}{c}0,09 * \\
(1,83)\end{array}$ & $\begin{array}{l}0,15^{* * *} \\
(3,00)\end{array}$ & $\begin{array}{l}0,15^{* * * *} \\
(3,02)\end{array}$ & $\begin{array}{c}0,04 \\
(0,93)\end{array}$ & $\begin{array}{c}0,06 \\
(1,44)\end{array}$ & $\begin{array}{c}-0,01 \\
(-0,24)\end{array}$ & $\begin{array}{c}-0,07 \\
(-1,44)\end{array}$ \\
\hline Alter & $\begin{array}{c}-0,02 \\
(-0,29)\end{array}$ & $\begin{array}{l}0,21 * * * \\
(3,04)\end{array}$ & $\begin{array}{c}-0,03 \\
(-0,41)\end{array}$ & $\begin{array}{c}0,02 \\
(0,26)\end{array}$ & $\begin{array}{l}-0,45 * * * \\
(-6,93)\end{array}$ & $\begin{array}{l}-0,45^{* * * *} \\
(-7,26)\end{array}$ & $\begin{array}{l}-0,40 * * * \\
(-6,80)\end{array}$ & $\begin{array}{c}0,10 \\
(1,36)\end{array}$ \\
\hline Geschlecht & $\begin{array}{c}-0,02 \\
(-0,50)\end{array}$ & $\begin{array}{c}-0,06 \\
(-1,31)\end{array}$ & $\begin{array}{c}0,02 \\
(0,35)\end{array}$ & $\begin{array}{c}0,04 \\
(0,78)\end{array}$ & $\begin{array}{l}0,10^{* *} \\
(2,55)\end{array}$ & $\begin{array}{c}0,03 \\
(0,72)\end{array}$ & $\begin{array}{c}0,04 \\
(1,01)\end{array}$ & $\begin{array}{c}-0,02 \\
(-0,35)\end{array}$ \\
\hline $\begin{array}{l}\text { Korrigiertes } \\
\mathrm{R}^{2}\end{array}$ & 0,31 & 0,27 & 0,14 & 0,20 & 0,34 & 0,42 & 0,48 & 0,24 \\
\hline $\mathrm{N}$ & 428 & 428 & 428 & 428 & 428 & 428 & 428 & 428 \\
\hline
\end{tabular}

$* \mathrm{p}<0,1 ; * * \mathrm{p}<0,05 ; * * * \mathrm{p}<0,01$. Es wurden die standardisierten Regressionskoeffizienten (in Klammern die t-Werte) angegeben.

Betrachtet man die Resultate im Hinblick auf die ersten beiden Hypothesen, so muß der darin formulierte, starke Erklärungsanspruch zurückgewiesen werden. Die kulturellen Präferenzen und die Handlungsrestriktionen der Akteure tragen zwar in allen Fällen zur statistischen Erklärung der jeweiligen abhängigen Variablen bei, doch nur in zwei von elf Fällen haben die Kontrollvariablen keinen statistisch signifikanten Einfluß. Dieses Resultat spricht dafür, daß die vorliegende theoretische Konzeption zwar durchaus durch die empirischen Ergebnisse unterstützt wird, daß aber die relevanten kulturellen Präferenzen und Handlungsrestriktionen der Akteure noch nicht vollständig spezifiziert sind. Betrachtet man daher im Einzelnen die Ergebnisse für die Kontrollvariablen, so ergibt sich das folgende Bild: geschlechtsspezifische Unterschiede jenseits der kulturellen Präferenzen und Handlungsrestriktionen lassen sich nur für zwei abhängige Variablen feststellen. Männer besitzen häufiger ein Auto und gehen häufiger in Kneipen. Dagegen finden sich schon in vier Fällen Einflüsse des Alters auf die abhängigen Variablen. Dabei zeigen sich drei starke Effekte auf Freizeitaktivitäten, die eher typisch für jüngere Altersgruppen sind: Kino-, Kneipen- und Diskothekenbesuch. Vor dem Hintergrund des theoretischen Modells hätte in diesen drei Fällen erwartet werden können, daß vor allem das Spannungsschema diese Aktivitäten beeinflußt, doch bleiben hier die statistischen Effekte vergleichsweise klein. Dies spricht sehr deutlich dafür, daß die hier vorgenommene Operationalisierung des Spannungsschemas allein nicht 
ausreicht, um die Ausübung dieser kulturellen Aktivitäten zu erklären, so daß mögliche Erweiterungen diskutiert werden sollten. Einen weiteren, aber deutlich kleineren Einfluß des Alters kann man darüber hinaus beim Ballettbesuch feststellen, wobei hier die älteren Jahrgänge zu häufigerem Besuch neigen. Zum Abschluß können noch die Ergebnisse für die dritte Kontrollvariable, Bildung, betrachtet werden. Hier können insgesamt sechs statistisch signifikante Effekte festgestellt werden. Erstens kann bei allen vier außerhalb des Haushalts ausgeübten hochkulturellen Praktiken ein gewisser, positiver Bildungseffekt beobachtet werden, während dieser beim Hören von Opernmusik im eigenen Haushalt nicht auftritt. Offensichtlich reicht hier die alleinige hochkulturelle Präferenz nicht aus, um den Besuch von Veranstaltungen dieser Art zu erklären. Darüber hinaus kann bei zwei der drei betrachteten Konsumgüter, beim Urlaub und beim Auto, ein positiver Einfluß der Bildung auf deren Besitz festgestellt werden. Insgesamt zeigen sich damit bei der Analyse der Kontrollvariablen relativ deutliche Muster, die die Suche nach kulturellen Präferenzen und Handlungsrestriktionen, die in das theoretische Modell zu integrieren wären, anleiten können.

Tabelle 5: Die Bedeutung von kulturellen Präferenzen und Handlungsrestriktionen für die Verfügung über Konsumgüter

\begin{tabular}{|c|c|c|c|c|c|c|}
\hline Variablen & Urlaub & & Delikatessen & & Autobesitz & \\
\hline Konstante & $\begin{array}{l}0,13 * * \\
(3,96)\end{array}$ & - & $\begin{array}{l}0,14 * * \\
(5,02)\end{array}$ & - & $\begin{array}{l}0,12^{* *} \\
(5,61)\end{array}$ & - \\
\hline Hochkultur & $\begin{array}{c}1,27 \\
(2,61)\end{array}$ & 0,007 & $\begin{array}{l}1,50 * * * \\
(9,09)\end{array}$ & 0,021 & $\begin{array}{c}0,89 \\
(0,78)\end{array}$ & 0,002 \\
\hline Spannung & $\begin{array}{c}0,76 \\
(1,80)\end{array}$ & 0,005 & $\begin{array}{c}1,00 \\
(0,00)\end{array}$ & 0,0 & $\begin{array}{l}1,55^{* *} \\
(6,46)\end{array}$ & 0,016 \\
\hline Trivialkultur & $\begin{array}{c}0,93 \\
(0,16)\end{array}$ & 0,0004 & $\begin{array}{l}0,69 * * \\
(4,71)\end{array}$ & 0,01 & $\begin{array}{c}1,14 \\
(0,67)\end{array}$ & 0,002 \\
\hline Einkommen & $\begin{array}{c}1,002 * * * \\
(31,13)\end{array}$ & 0,12 & $\begin{array}{c}1,002 * * * \\
(29,74)\end{array}$ & 0,086 & $\begin{array}{c}1,001 * * * \\
(19,74)\end{array}$ & 0,06 \\
\hline Bildung & $\begin{array}{l}1,89 * * * \\
(13,57)\end{array}$ & 0,04 & $\begin{array}{c}0,92 \\
(0,42)\end{array}$ & 0,001 & $\begin{array}{c}1,26^{*} \\
(2,83)\end{array}$ & 0,007 \\
\hline Alter & $\begin{array}{c}0,99 \\
(0,72)\end{array}$ & 0,002 & $\begin{array}{c}1,00 \\
(0,02)\end{array}$ & 0,0 & $\begin{array}{c}0,99 \\
(1,62)\end{array}$ & 0,004 \\
\hline Geschlecht & $\begin{array}{c}0,95 \\
(0,03)\end{array}$ & 0,0001 & $\begin{array}{c}1,50 \\
(2,36)\end{array}$ & 0,005 & $\begin{array}{l}1,63 * \\
(3,37)\end{array}$ & 0,008 \\
\hline $\begin{array}{l}\text { Korrigiertes } \\
\text { Pseudo- } \mathrm{R}^{2}\end{array}$ & 0,24 & & 0,15 & & 0,14 & \\
\hline $\mathrm{N}$ & 311 & & 311 & & 323 & \\
\hline
\end{tabular}

$* \mathrm{p}<0,1 ; * * \mathrm{p}<0,05 ; * * * \mathrm{p}<0,01$. Es wurden die Effektparameter (in Klammern die Wald-Statistik) angegeben. In der rechten Spalte wurde jeweils die Veränderung des Pseudo- $\mathrm{R}^{2}$ durch die jeweilige Variable angegeben.

Betrachtet man nun die Ergebnisse für die kulturellen Präferenzen im Einzelnen, so zeigen sich die folgenden Muster: je stärker die Hochkulturorientierung, desto häufiger besuchen die Personen das Gewandhaus, Ballettaufführungen, das Schauspielhaus und das Museum der bildenden Künste. Zudem hören diese Personen häufiger Opernmusik und konsumieren eher Delikatessen. Einen negativen Einfluß übt die Hochkulturorientierung auf den Besuch von Diskotheken aus. Es kann weiterhin festgestellt werden, daß Personen mit stärkerer Ori- 
entierung am Spannungsschema öfter Kneipen, Cafés, Kinos und Diskotheken besuchen und zudem eher ein Auto besitzen. Dagegen findet man diese Personen seltener im Gewandhaus und beim Hören von Opernmusik. Beim Trivialschema kann schließlich kein positiver Einfluß festgestellt werden. Dagegen finden sich einige negative Effekte desselben: Je stärker diese kulturelle Präferenz ist, desto seltener besuchen die Personen das Ballett, das Schauspielhaus, das Museum der bildenden Künste, Kneipen und Cafés, und Kinos. Zudem konsumieren diese Personen seltener Delikatessen. Zusammenfassend kann für die inhaltliche Wirkungsrichtung der Schemata festgehalten werden, daß diese Variablen - über die Musikvorlieben operationalisiert - die Häufigkeit von Aktivitäten und den Besitz von Konsumgütern positiv und deutlich beeinflussen, die zur gleichen Zeichengruppe gehören. Das Spannungsschema und das Hochkulturschema weisen dagegen nur wenige negative Einflüsse auf, während das Trivialschema mit eindeutigen Abneigungen gegen zahlreiche der hier berücksichtigten Freizeitpraktiken und Konsumformen vor allem aus dem hochkulturellen und spannungsorientierten Bereich verbunden ist.

Insgesamt muß aber überraschen, daß die relativ unspezifischen kulturellen Präferenzen, die zudem sehr sparsam operationalisiert wurden, derartig viele und relativ starke statistische Effekte auf die abhängigen Variablen ausüben. Angesichts der Diskussionslage in der Sozialpsychologie (Frey/Stahlberg/Gollwitzer 1993), die generell von einem schwachen Zusammenhang von allgemeinen Einstellungen und Verhalten ausgeht, muß hier festgestellt werden, daß Schulze mit den drei alltagsästhetischen Schemata ausgesprochen erklärungskräftige kulturelle Präferenzen konzipiert hat. In Hypothese 3 wurde behauptet, daß deren Erklärungskraft für musiknahe und musikferne Aktivitäten gleich groß sein sollte, auch wenn die Operationalisierung allein auf der Basis des Musikgeschmacks vorgenommen wurde. Diese These findet nur eine begrenzte Unterstützung. Die durchschnittliche Höhe des Betrags der standardisierten Regressionskoeffizienten der kulturellen Präferenzen bei den musiknahen Praktiken liegt bei 0,18, während sie bei den musikfernen Aktivitäten den Wert von 0,13 erreicht. Es kann also insgesamt eine bessere Erklärungskraft bei den musiknahen Praktiken festgestellt werden, so daß bei der zukünftigen Operationalisierung von kulturellen Präferenzen der Musikgeschmack zwar einen zentralen Aspekt darstellen, doch durch weitere Bereiche des Geschmacks ergänzt werden sollte.

Nachdem die Ergebnisse der statistischen Analysen in Tabelle 4 und 5 im Hinblick auf die ersten drei Hypothesen betrachtet wurden, wenden wir uns nun den auf die Opportunitätsstrukturen bzw. Handlungsrestriktionen bezogenen Thesen zu. Hypothese 4 behauptet, daß die Entfernung des Wohnstandortes vom Stadtzentrum nur bei Aktivitäten relevant sein sollte, die im Stadtzentrum konzentriert sind. Diese Aussage findet in den Analysen eine deutliche Unterstützung. Bei vier von fünf im Stadtzentrum konzentrierten kulturellen Angeboten (Gewandhaus, Ballett, Museum, Kneipen/Cafés) hat die Entfernung der Wohnung vom Stadtzentrum einen negativen Einfluß auf die Häufigkeit der Ausübung der jeweiligen Aktivität. Dagegen besitzt diese Handlungsrestriktionen bei den über die Stadt verteilten Angeboten (Kino, Diskotheken) keinerlei Einfluß. Damit wird eindringlich die von vielen Stadtsoziologen vertretene These der Bedeutung von räumlichen Voraussetzungen für das Ausleben von Lebensstilen unterstützt (Dangschat 1994; Zum Felde/Alisch 1992; Blasius 1993). Für die Stadt Leipzig wird sehr deutlich, daß die Nähe der Wohnung zur Innenstadt die Gestaltung eines hochkulturellen oder spannungsorientierten Lebensstils in der Öffentlichkeit erleichtert. Freilich muß dabei berücksichtigt werden, daß die Wahl eines Wohnstandortes durchaus auch von kulturellen Präferenzen geprägt ist (Schneider/Spellerberg 1999). Als Konsequenz dieser früheren, zum Teil präferenzgesteuerten Entscheidung entstehen dann aber neue Handlungsrestriktionen, die in späteren Entscheidungsprozessen den Handlungsspielraum einschränken können. Damit wird insgesamt noch einmal deutlich, daß eine dringende Aufgabe der Forschung in diesem Bereich der Sozialstrukturanalyse die Längsschnitt- 
analyse des Zusammenspiels von äußeren Handlungsrestriktionen und kulturellen Vorlieben im Lebensverlauf sein müßte (Lüdtke 1989; Hartmann 1999).

Wenden wir uns schließlich noch der fünften Hypothese mit ihren drei Unterhypothesen zu. Generell wird hier behauptet, daß die kulturellen Präferenzen vor allem in Niedrigkostensituationen relevant, während in Hochkostensituationen in stärkerem Maße die Handlungsrestriktionen erklärungskräftig sind. In der ersten Unterhypothese wurde diese These für Situationen mit extrem niedrigen Kosten spezifiziert. Hier sollten alle Handlungsrestriktionen irrelevant sein. Dies wurde am Beispiel des Hörens von Opernmusik im eigenen Haushalt überprüft. Tatsächlich kann für diese kulturelle Aktivität festgestellt werden, daß keine der Handlungsrestriktionen einen statistisch signifikanten Einfluß aufweist. Damit erfährt diese Hypothese deutliche Unterstützung durch die empirischen Resultate. Die zweite Unterhypothese der fünften Hypothese behauptete für die Handlungssituationen mit geringen Kosten, also für die außer Haus ausgeübten kulturellen Aktivitäten, daß die kulturellen Präferenzen eine stärkere Erklärungskraft besitzen als die Handlungsrestriktionen. Letztere sollten aber in diesen Situationen das Verhalten beeinflussen. Auch diese These kann durch die empirischen Resultate gestützt werden. Während in diesen Modellen jeweils durchschnittlich zwei kulturelle Präferenzen einen statistisch signifikaten Einfluß aufweisen, gilt dies durchschnittlich nur für 1,1 Handlungsrestriktionen. Zudem ist auch die Höhe des durchschnittlichen Betrags der standardisierten Regressionskoeffizienten für die Handlungsrestriktionen mit 0,07 deutlich niedriger als für die kulturellen Präferenzen mit 0,14 . Schließlich können wir noch die dritte Unterhypothese der fünften Hypothese betrachten, die eine stärkere Erklärungskraft der Handlungsrestriktionen in Hochkostensituationen vorhersagt. Da es sich bei den abhängigen Variablen um die Verfügung über spezifische Konsumgüter handelt, wurde als Handlungsrestriktion nur das verfügbare Einkommen der befragten Personen berücksichtigt. Bei diesen drei Modellen zeigt sich, daß durchschnittlich nur noch eine der drei kulturellen Präferenzen einen statistisch signifikanten Einfluß aufweist. Darüber hinaus kann aus den Werten für die Veränderung von Pseudo- $\mathrm{R}^{2}$ durch die einzelnen Variablen gefolgert werden, daß hier das verfügbare Einkommen den dominanten Einfluß ausübt. Insofern kann auch die dritte Unterhypothese von Hypothese 5 bestätigt werden. Interessant ist allerdings, daß selbst in diesen Hochkostensituationen die kulturellen Präferenzen zum Teil noch entscheidungsrelevant sind. Dies spricht wiederum für die eigenständige Berücksichtigung der kulturellen Sphäre bei der Erklärung von sozialem Handeln, wie es von der Lebensstilforschung nahegelegt wurde. Freilich wird hier aber auch deutlich, daß in Hochkostensituationen die Bedeutung von materiellen Ressourcen eine größere explanatorische Rolle einnimmt. Da diese aber in stärkerem Maße von der Klassen- bzw. Schichtstruktur einer Gesellschaft geprägt sind, wird diese vor allem in Hochkostensituationen von größerer Relevanz sein, also häufig in Situationen in denen es um die langfristigen Lebenslagen und Lebenschancen von Personen geht.

\section{Zusammenfassung}

Die Diskussion in der Sozialstrukturanalyse war in den vergangenen zwanzig Jahre durch den Gegensatz zwischen den Konzepten Klasse und Schicht auf der einen Seite und den Begriffen sozialen Milieus und Lebensstile auf der anderen Seite gekennzeichnet. Die letztgenannten Konzepte wurden in die Debatte eingeführt, um die sozialen Wandlungsprozesse der vergangenen Jahrzehnte und die damit verbundene relative Abkoppelung der Lebensstile von den materiellen Lebensbedingungen erfassen zu können und die Sozialstrukturanalyse näher an das soziale Handeln der Menschen heranzuführen. Dagegen wurde hier die These vertreten, daß in kapitalistischen Industriegesellschaften schon seit Beginn des 20. Jahrhunderts keine Deckung zwischen Klassen, Milieus und Lebensstilen bestanden hat. Damit ist eine Ersetzung von Klassen- oder Schichtkonzepten durch Milieu- oder Lebensstilbegriffe zur deskriptiven Erfassung der Sozialstruktur der Gesellschaft über historische Wandlungs- 
prozesse kaum begründbar. Zudem wurde deutlich gemacht, daß das Lebensstilkonzept bei der Erklärung von sozialem Handeln auf vergleichbare Probleme stößt wie der Klassenbegriff, da es sich in beiden Fällen lediglich um Klassifikationen von Personen handelt, die aber keinen sozialen Mechanismus enthalten, der das sozialen Handeln von Menschen theoretisch erklärt. Während nun aber in der neueren Klassentheorie ein Brückenschlag zu systematischen Handlungstheorien vorgenommen wurde (Wright 1997; Becker 2000; Breen/ Goldthorpe 1997), verbleibt die Lebensstilforschung dagegen häufig im Bereich der Variablensoziologie.

Als alternatives Konzept zur Ersetzung des Lebensstilbegriffs wurde daher der Begriff der kulturellen Präferenzen eingeführt, der sich in systematischer Weise auf gängige Theorien des sozialen Handelns beziehen läßt. Zudem besitzt er eine sehr hohe inhaltliche Übereinstimmung mit dem Lebensstilbegriff, freilich mit dem entscheidenden Unterschied, daß er sich nicht auf typische Muster sozialen Handelns bezieht, sondern auf die Vorliebe für bestimmte Klassen sozialen Handelns, so daß er zur systematischen Erklärung von Verhalten verwendet werden kann. Hier wurde daher in exemplarischer Weise ein Modell zielgerichteten Handelns skizziert, das das Verhalten von Personen unter Bezug auf ihre jeweiligen Präferenzen und ihre Opportunitätsstuktur erklärt, so daß die kulturellen Präferenzen in das Modell integriert werden können. Der Begriff der Opportunitätsstruktur bzw. der Handlungsrestriktionen ergibt sich aus dem Verhältnis von zwei Konzepten: erstens den Kosten für bestimmte Handlungen und zweitens durch die Ressourcen, die dem Akteur zur Verfügung stehen. In Anschluß an die Debatte über Situationen mit unterschiedlichen Kostenstrukturen in der Soziologie wurde hier die These vertreten, daß die kulturellen Präferenzen vor allem in Niedrigkostensituationen handlungserklärend sind, während in Hochkostensituationen die Handlungsrestriktionen stärker greifen. Daher können an dieser Stelle auch Begriffe wie Klasse und Schicht mit der vorgestellten handlungstheoretischen Perspektive verknüpft werden, da die handlungsrelevanten Ressourcen zum Teil klassen- oder schichtspezifisch verteilt sind. Dies gilt zum Teil auch für die Kosten von bestimmten Handlungen. Insofern ist die Zugehörigkeit zu bestimmten Klassen oder Schichten vor allem in Hochkostensituationen von Bedeutung, wenn also die Verfügung über materielle Ressourcen im Vordergrund steht, während die kulturellen Präferenzen als Nachfolger des Lebensstilbegriffs vor allem in Niedrigkostensituationen zentral sind. Freilich wird man schon an dieser Stelle zu einer Ausweitung der Perspektive gelangen, da Handlungsresourcen und -kosten nicht nur klassen- und schichtenspezifisch verteilt sind, sondern sich auch nach Geschlecht, Alter und ethnischer Zugehörigkeit unterscheiden (vgl. Kreckel 1992). An die vorgestellte handlungstheoretische Konzeption lassen sich also sowohl die traditionellen Klassen- und Schichtkonzepte anknüpfen, wie auch der gegenwärtig stark diskutierte Lebensstilbegriff. Zudem kann eine theoretisch präzisere Verbindung zwischen den strukturellen Bedingungen des Handelns und den Präferenzen von Akteuren hergestellt werden: einerseits werden die Vorlieben und Dispositionen von Personen durch ihre jeweilige strukturelle Lage geprägt, andererseits können die Akteure ihre Präferenzen jeweils nur unter Berücksichtigung der Opportunitätsstrukturen verwirklichen. Gerade am Beispiel des Wohnstandortes und der damit verbundenen räumlichen Distanz zu Kultureinrichtungen konnte allerdings verdeutlicht werden, daß gegebene Handlungsrestriktionen durchaus die Folgen von präferenzgesteuerten Entscheidungen in der Vergangenheit sein können.

Das skizzierte theoretische Konzept wurde auf der Basis von zwei, in Leipzig durchgeführten schriftlichen Umfragen empirisch überprüft. Dabei wurde deutlich, daß man auf der Basis der kulturellen Präferenzen und der Opportunitätsstrukturen der befragten Personen die kulturellen Aktivitäten und die Verfügung über Konsumgüter mit durchaus zufriedenstellenden Ergebnissen erklären kann. Vor allem für die kulturellen Präferenzen muß festgestellt werden, daß diese als relativ unspezifische Einstellungen, die zudem höchst sparsam operationalisiert wurden, eine beachtliche Erklärungsleistung aufweisen. Dies spricht sehr deutlich 
dafür, daß die von der Lebensstilforschung reklamierte Eigenbedeutung der kulturellen Sphäre für das soziale Handeln nicht vernachlässigt werden kann. Freilich ergab sich in der empirischen Studie, daß die hier vorgenommene Operationalisierung der kulturellen Präferenzen über den Musikgeschmack hinaus erweitert werden muß, da die Erklärungsleistung für musiknahe Aktivitäten höher lag als für musikferne Aktivitäten.

Neben den kulturellen Präferenzen und den Handlungsrestriktionen wurden in der empirischen Studie auch das Alter, das Geschlecht und die Bildung der Befragten als Kontrollvariablen berücksichtigt, da diese Variablen in der bisherigen Forschung die stärkste Prägekraft im Hinblick auf Lebensstile gezeigt haben. Entgegen der aus dem skizzierten theoretischen Modell abgeleiteten Hypothese ergaben sich für diese Kontrollgrößen statistisch signifikante Effekte auf die abhängigen Variablen. Dabei fanden sich drei inhaltliche Muster, die Hinweise für eine weitere Spezifikation des hier zugrundegelegten handlungstheoretischen Modells enthalten. Erstens zeigte sich, daß Männer häufiger Kneipen besuchen und eher ein Auto besitzen als Frauen, also ein geschlechtsspezifisches Muster. Zweitens ergab sich als dominantes Ergebnis im Hinblick auf altersspezifische Unterschiede, daß außerhäusliche Aktivitäten wie Kino-, Kneipen- und Diskothekenbesuch viel schwächer als erwartet durch das Spannungsschema erklärt werden konnten und dagegen starke Einflüsse des Alters feststellbar waren. Gerade an diesem Beispiel wird deutlich, daß eine stärkere Spezifikation von kulturellen Präferenzen und Handlungsrestriktionen notwendig ist. Schließlich zeigt sich drittens im Hinblick auf die Bildung als dritte Kontrollvariable, daß diese auf alle außerhäuslichen hochkulturellen Aktivitäten einen positiven Effekt ausübt. Auch hier müßte offensichtlich über eine stärkere Präzisierung von kulturellen Präferenzen und Handlungsrestriktionen nachgedacht werden, wobei aber wiederum das Muster der Effekte eine deutliche Suchrichtung vorgibt.

Schließlich wurde im Anschluß an die skizzierte Unterscheidung zwischen Niedrig- und Hochkostensituationen die relative Erklärungskraft von kulturellen Präferenzen und Handlungsrestriktionen untersucht. Dabei wurde deutlich, daß in Situationen mit sehr niedrigen Kosten allein die kulturellen Präferenzen relevant sind, in Situationen mit etwas höheren Kosten war diese Dominanz der kulturellen Vorlieben geringer ausgeprägt. Hier griffen bei der Erklärung der meisten Freizeitaktivitäten auch die Handlungsrestriktionen, allerdings mit statistisch geringeren Einflüssen. Hier wurde auch bestätigt, daß die räumliche Struktur der kulturellen Angebote einen Einfluß auf die individuelle Nutzungshäufigkeit besitzt. Schließlich konnte für Situationen mit vergleichsweise hohen Kosten gezeigt werden, daß hier die Handlungsrestriktionen, wobei hier nur die Verfügung über ökonomische Ressourcen berücksichtigt wurde, eine zentrale explanatorische Rolle einnehmen. Allerdings hatten selbst in diesen Hochkostensituationen die kulturellen Präferenzen teilweise statistisch signifikante Effekte, was wiederum für deren Bedeutung bei der Handlungserklärung spricht.

Damit ist insgesamt deutlich geworden, daß ein zentraler Begriff der gegenwärtigen Diskussion in der Sozialstrukturanalyse mit leichten konzeptuellen Modifikationen in ein - hier freilich ausgesprochen sparsam konzipiertes - handlungstheoretisches Konzept integriert werden kann. Eine derartige Verknüpfung mit Konzepten zur Erklärung sozialen Handelns könnte die theoretische und empirische Leistungsfähigkeit des Lebensstilbegriffs bzw. des an seine Stelle gesetzten Konzepts der kulturellen Präferenzen deutlich erhöhen. Zudem bietet die vorgeschlagene Strategie der Unterscheidung von Niedrig- und Hochkostensituationen auch die Möglichkeit, andere theoretische Konzepte der sozialen Ungleichheit, wie den Klassenbegriff, der zentral für die Verteilung von ökonomischen Ressourcen ist, in die theoretische Konzeption zu integrieren. Damit ist ein fruchtbarer Weg für eine Verknüpfung von Sozialstrukturanalyse und Handlungstheorie angedeutet, der die Grundbegriffe der Sozialstrukturanalyse tatsächlich enger an das soziale Handeln der Akteure heranführen könnte und zudem diese verschiedenen Konzepte integriert und nicht gegeneinander ausspielt. 


\section{Literatur}

Adam, Thomas (1999): Arbeitermilieu und Arbeiterbewegung in Leipzig 1871 - 1933. Köln: Böhlau.

Beck, Ulrich (1986): Risikogesellschaft. Auf dem Weg in eine andere Moderne. Frankfurt: Suhrkamp.

Becker, Rolf (2000): Klassenlage und Bildungsentscheidungen. Eine empirische Anwendung der WertErwartungstheorie, in: Kölner Zeitschrift für Soziologie und Sozialpsychologie 52: 450 - 474.

Blasius, Jörg (1993): Gentrification und Lebensstile. Eine empirische Untersuchung. Wiesbaden: DUV.

Bögenhold, Dieter (1994): Soziologie der Lebenstile: Substitution oder Ergänzung in der Tradition der sozialstratifikatorischen Forschung? in: Schweizerische Zeitschrift für Soziologie 20: 439 - 459.

Bourdieu, Pierre (1982): Die feinen Unterschiede. Kritik der gesellschaftlichen Urteilskraft. Frankfurt: Suhrkamp.

Bourdieu, Pierre/Wacqant, Loic (1996): Reflexive Anthropologie. Frankfurt: Suhrkamp.

Breen, Richard/Goldthorpe, John H. (1997): Explaining Educational Differentials, in: Rationality and Society 9: $275-305$.

Collins, Randall/Hanneman, Robert A. (1998): Modelling the Interaction Ritual Theory of Solidarity, in: Patrick Doreian/Thomas Fararo (Hrsg.): The Problem of Solidarity. Theories and Models. New York: Gordon and Breach, 213 - 237.

Dangschat, Jens (1994): Lebensstile in der Stadt. Raumbezug und konkreter Ort von Lebensstilen und Lebensstilisierungen, in: Jens Dangschat/Jörg Blasius (Hrsg.): Lebensstile in den Städten. Konzepte und Methoden. Opladen: Leske + Budrich, 335-354.

Diekmann, Andreas (1995): Empirische Sozialforschung. Grundlagen, Methoden, Anwendungen. Reinbek: Rowohlt.

Diekmann, Andreas (1996): Homo ÖKOnomicus. Anwendungen und Probleme der Theorie rationalen Handelns im Umweltbereich, in: Andreas Diekmann/Carlo C. Jaeger (Hrsg.): Umweltsoziologie. Sonderheft 36 der Kölner Zeitschrift für Soziologie und Sozialpsychologie. Opladen: Westdeutscher Verlag, $89-118$.

Elster, Jon (1989): Nuts and Buts for the Social Sciences. Cambridge: Cambridge University Press.

Esser, Hartmut (1996): What is Wrong with „Variable Sociology“"? in: European Sociological Review 12: $159-166$.

Esser, Hartmut (2002): In guten wie in schlechten Tagen? Das Framing der Ehe und das Risiko zur Scheidung, in: Kölner Zeitschrift für Soziologie und Sozialpsychologie 54: 27 - 63.

Fine, Gary A. (Hrsg.) (1995): A Second Chicago School? The Development of a Postwar American Sociology. Chicago: University of Chicago Press.

Franz, Peter (1986): Der „Constrained Choice”-Ansatz als gemeinsamer Nenner individualistischer Ansätze in der Soziologie, in: Ein Vorschlag zur Integration. Kölner Zeitschrift für Soziologie und Sozialpsychologie 38: $32-54$.

Frey, Dieter, Stahlberg, Dagmar und Peter Gollwitzer (1993): Einstellung und Verhalten. Die Theorie des überlegten Handelns und die Theorie des geplanten Verhaltens, in: Dieter Frey/Martin Irle (Hrsg.): Kognitive Theorien der Sozialpsychologie. Bern: Huber, $361-398$.

Ganzeboom, Harry (1987): Cultural Audience Formation in the Netherlands Between 1962 and 1983, in: Douglas Shaw/William S. Hendon/C. Richard Waits (Hrsg.): Artists and Cultural Consumers. Akron: Association for Cultural Economics, 179-191.

Gebesmair, Andreas (2001): Grundzüge einer Soziologie des Musikgeschmacks. Wiesbaden: Westdeutscher Verlag.

Gerhards, Jürgen/Rössel, Jörg (2002): Lebensstile und ihr Einfluß auf das Ernährungsverhalten von Jugendlichen, in: Soziale Welt 53: 261 - 284.

Greene, William H. (1992): Econometric Analysis. Upper Saddle River: Prentice Hall.

Hartmann, Peter H. (1999): Lebensstilforschung. Darstellung, Kritik und Weiterentwicklung. Opladen: Leske + Budrich. 
Hedström, Peter/Swedberg, Richard (1998): Social Mechanisms: An Introductory Essay, in: Peter Hedström/Richard Swedberg (Hrsg.): Social Mechanisms. An Analytical Approach to Social Theory. Cambridge: Cambridge University Press, $1-31$.

Karl H. Hörning/Michailow, Matthias (1990): Lebensstil als Vergesellschaftungsform: Zum Wandel von Sozialstruktur und sozialer Integration, in: Peter A. Berger/Stefan Hradil (Hrsg.): Lebenslagen, Lebensstile, Lebensläufe. Sonderband 7 der Sozialen Welt. Göttingen: Schwartz, 501 - 521.

Kreckel, Reinhard (1992): Politische Soziologie der sozialen Ungleichheit. Frankfurt: Campus.

Hradil, Stefan (1987): Sozialstrukturanalyse in einer fortgeschrittenen Gesellschaft. Opladen: Leske + Budrich.

Hradil, Stefan (1999): Soziale Ungleichheit in Deutschland. Opladen: Leske + Budrich.

Hradil, Stefan (2001): Eine Alternative? Einige Anmerkungen zu Thomas Meyers Aufsatz „Das Konzept der Lebensstile in der Sozialstrukturforschung “, in: Soziale Welt 52: 273 - 282.

Joas, Hans (1992): Die Kreativität des Handelns. Frankfurt: Suhrkamp.

Kocka, Jürgen (1990): Arbeitsverhältnisse und Arbeiterexistenzen. Grundlagen der Klassenbildung im 19. Jahrhundert. Bonn: Dietz.

Konietzka, Dirk (1995): Lebensstile im sozialstrukturellen Kontext. Opladen: Westdeutscher Verlag.

Lechner, Götz (1998): Ist die Erlebnisgesellschaft in Chemnitz angekommen? in: Peter A. Berger/Michael Vester (Hrsg.): Alte Ungleichheiten, Neue Spaltungen. Opladen: Leske + Budrich, 257 - 274.

Lüdtke, Hartmut (1989): Expressive Ungleichheit. Zur Soziologie der Lebensstile. Opladen: Leske + Budrich.

Lüdtke, Hartmut (1990): Lebensstile als Dimension handlungsproduzierter Ungleichheit, in: Peter A. Berger/Stefan Hradil (Hrsg.): Lebenslagen, Lebensstile, Lebensläufe. Sonderband 7 der Sozialen Welt. Göttingen: Schwartz, 433 - 454.

Lüdtke, Hartmut (1995): Zeitverwendung und Lebensstile. Münster: Lit.

McKinnon, Neil J. (1994): Symbolic Interactionism as Affect Control. Albany: SUNY.

Mensch, Kirsten (2000): Niedrigkostensituationen, Hochkostensituationen und andere Situationstypen: Ihre Auswirkungen auf die Möglichkeit von Rational-Choice-Erklärungen, in: Kölner Zeitschrift für Soziologie und Sozialpsychologie 52: $256-270$.

Meyer, Thomas (2001): Das Konzept der Lebensstile in der Sozialstrukturforschung - eine kritische Bilanz, in: Soziale Welt 52: $255-271$.

Mooser, Josef (1984): Arbeiterleben in Deutschland 1900 - 1970. Klassenlagen, Kultur und Politik. Frankfurt: Suhrkamp.

Müller, Hans-Peter (1986): Kultur, Geschmack und Distinktion. Grundzüge der Kultursoziologie Pierre Bourdieus, in: Friedhelm Neidhardt/M. Rainer Lepsius/Johannes Weiß (Hg.): Kultur und Gesellschaft. Sonderheft 27 der Kölner Zeitschrift für Soziologie und Sozialpsychologie. Opladen: Westdeutscher Verlag, 162-190.

Müller, Hans-Peter (1992): Sozialstruktur und Lebensstile. Der neuere theoretische Diskurs über soziale Ungleichheit. Frankfurt: Suhrkamp.

Müller, Walter (1998): Parteiensystem und Klassenstruktur, in: Kölner Zeitschrift für Soziologie und Sozialpsychologie 50: 1 - 45.

Müller-Schneider, Thomas (1994): Von Schichten zu Erlebnismilieus. Der Wandel der Milieustruktur in der Bundesrepublik Deutschland. Wiesbaden: DUV.

Müller-Schneider, Thomas (1996): Wandel der Milieulandschaft in Deutschland. Von hierarchisierenden zu subjektorientierten Wahrnehmungsmustern, in: Zeitschrift für Soziologie 25: 190 - 206.

Opp, Karl-Dieter (1999): Contending Conceptions of the Theory of Rational Action, in: Journal of Theoretical Politics 11: $171-202$.

Otte, Gunnar (2000): Urlaub als lebensführungsspezifisches Investitionsverhalten, in: Tourismus Journal 4: $471-499$. 
Pierenkemper, Toni (1987): The Standard of Living and Employment in Germany, 1850 - 1980: An Overview, in: Journal of European Economic History 16: 51 - 74.

Quandt, Markus/Ohr, Dieter (2004): Worum geht es, wenn es um nichts geht? Zum Stellenwert von Niedrigkostensituationen in der Rational-Choice Modellierung sozialen Handelns, erscheint in: Kölner Zeitschrift für Soziologie und Sozialpsychologie.

Schneider, Nicole/Spellerberg, Annette (1999): Lebenstile, Wohnbedürfnisse und räumliche Mobilität. Opladen: Leske + Budrich.

Schulze, Gerhard (1992): Die Erlebnisgesellschaft. Kultursoziologie der Gegenwart. Frankfurt: Campus.

Spellerberg, Annette (1996): Soziale Differenzierung durch Lebensstile. Eine empirische Untersuchung zur Lebensqualität in West- und Ostdeutschland. Berlin: Sigma.

Sperber, Jonathan (1997): The Kaiser's Voters. Electors and Elections in Imperial Germany. Cambridge: Cambridge University Press.

Spree, Reinhard (1997): Klassen- und Schichtbildung im Medium des privaten Konsums: vom späten Kaiserreich in die Weimarer Republik, in: Historical Social Research 22: 29 - 80.

Stadt Leipzig (2001): Statistisches Jahrbuch 2001. Amt für Statistik und Wahlen: Leipzig.

Stadt Leipzig (2003): Kommunale Bürgerumfrage 2002. Amt für Statistik und Wahlen: Leipzig.

Uttitz, Pavel (1985): Determinanten des Freizeitverhaltens in den letzten 30 Jahren, in: ZA-Informationen 16: $22-39$.

Wright, Erik O. (1997): Class Counts. Comparative Studies in Class Analysis. Cambridge: Cambridge University Press.

Zerger, Frithjof (2000): Klassen, Milieus und Individualisierung. Eine empirische Untersuchung zum Umbruch der Sozialstruktur. Frankfurt: Campus.

Zintl, Reinhard (1989): Der Homo Oeconomicus: Ausnahmeerscheinung in jeder Situation oder Jedermann in Ausnahmesituationen? In: Analyse \& Kritik 11: 52 - 69.

Zum Felde, Wolfgang und Monika Alisch (1992): Zur Bedeutung des Raumes für Lebensbedingungen und Lebensstile von Bewohnern innenstadtnaher Nachbarschaften in Hamburg, in: Stefan Hradil (Hrsg.): Zwischen Bewußtsein und Sein. Opladen: Leske + Budrich, 173 - 194.

Dr. Jörg Rössel, Universität Leipzig, Institut für Kulturwissenschaften, Beethovenstr. 15, 04107 Leipzig roessel@rz.uni-leipzig.de 